\title{
Introduction to the Special Edition on Clinical and Educational Digital Interventions Via Technology
}

\author{
Elizabeth A. Krupinski ${ }^{1} \cdot$ Bradford L. Felker $^{2} \cdot$ Sarah E. Nasatir-Hilty ${ }^{3} \cdot$ Donald M. Hilty $^{4}$ (D) \\ Received: 31 July 2020 / Revised: 31 July 2020 / Accepted: 11 August 2020 / Published online: 22 August 2020 \\ (C) Springer Nature Switzerland AG 2020
}

\section{Introduction}

This special issue, "Evidence-Based Clinical and Educational Digital Interventions via Video, Mobile Health, Apps and Wearable Technologies", will help providers, faculty, residents, program directors, administrators, and other interprofessional partners improve clinical care, training, and leadership at the interface of behavioral health and technology for culturally and otherwise diverse populations. When recruiting authors, we asked them to consider a variety of topics such as applying the evidence base on technology to clinical assessment, triage, consultation, and treatment of behavioral and mental disorders; skills, attitudes, and knowledge for providers to use technologies to help patients via evidence-based approaches; translating best research practices to the bedside and between healthcare systems; using implementation science methods to promote adoption and integration of evidence-based technology-based

JTIBS Special Edition on Clinical and Educational Interventions for Technologies

Donald M. Hilty

donh032612@gmail.com

Elizabeth A. Krupinski

ekrupin@emory.edu

Bradford L. Felker

Bradford.Felker@va.gov

Sarah E. Nasatir-Hilty

sarah.elizabeth.nasatir-hilty@emory.edu

1 Department of Radiology \& Imaging Sciences, Emory University, Atlanta, GA, USA

2 Department of Psychiatry \& Behavioral Sciences and Director Telemental Health, Puget Sound Veterans Affairs, University of Washington, Seattle, WA, USA

3 Nell Hodgson Woodruff School of Nursing of Emory University, Hills, GA, USA

4 Department of Psychiatry \& Behavioral Sciences, UC Davis \& VA N. California HCS, 10535 Hospital Way, Mather, CA 95655 (116/ SAC), USA practices, interventions and policies into routine health care and public health settings; and exploring the unintended consequences of monitoring and sensing technologies used for daily life, health, and health care. We purposely discouraged certain types of submissions: those simply on new apps that are "cool" or innovative, but not evaluated systematically; "good" ideas without demonstrated impact; "commercials" for publically available products; and case reports, editorials, and other papers that were not generally applicable to a variety of populations and settings.

The resulting papers fell into two sections. Section 1 covers research on the application of technology to practice. Section 2 covers educational interventions, competencies, and organizational leadership with respect to clinical, training, research, and professional development outcomes and assessment.

\section{Section 1}

The technology for practice section has 5 papers dealing with a range of important topics, with 2 review papers and 3 on Veteran populations, including one examining care manager contributions to teams related to technology. Connolly et al. provide a very nice exploration of key factors that influence the uptake and use of behavioral health smartphone applications (apps) in "Leveraging implementation science to understand factors influencing sustained use of behavioral health apps: a narrative review." They first provide a concise explanation of implementation science and the framework for the review. The findings suggest that apps should be simple and easy to use and must meet a clear need. The attitudes of users are critical and developers must pay attention to user beliefs about $\mathrm{mH}$ apps, training, resources for successful uptake, and context as implementation strategies may vary whether apps are marketed directly to consumers or hospital systems.

Hilty et al. report on "A scoping review of sensors, wearables and remote monitoring for behavioral health: uses, outcomes, clinical competencies and research directions." They assessed the use of devices to measure and assess 
physiological and behavioral data in real time in behavioral health and assess gaps related to methods, interventions, and outcomes. The included studies focused on mood, anxiety disorders, psychological stress/stress disorders, and healthy adults or college students. The results indicate that research is starting to use standardized methods, interventions, and evaluation measures, with longitudinal correlation, prediction, and biomarking of outcomes. However, these technologies also pose many challenges including quality, education/training, skills, technology, integration into workflow, and privacy, necessitating much more research and development of clinician and institutional competencies.

All three of the studies with Veterans highlight ways that technology (video-based telehealth specifically) can help care for this important population, including those in rural areas. Each study targeted a different diagnostic cohort of Veterans. Ecker et al. also studied in-home telehealth, assessing "The impact of co-occurring anxiety and alcohol use disorders on video telehealth utilization among rural Veterans." In this retrospective analysis, they examined data from a cohort of Veterans receiving at least one video-based behavioral health visit over a 3-year period. They used multilevel linear growth curve models to examine growth in telehealth use over time for Veterans with anxiety only, alcohol use disorder only, and co-occurring anxiety and alcohol use disorders. For each subsequent year, the percent of total video visits increased for all groups, but less so for the co-occurring group making them especially at risk for not receiving tele-care. They conclude that we need to improve ways to reach individuals with less access to telehealth through mobile health technologies.

Myers et al. also used a video-based system for treatment and summarize their "Lessons learned in implementing VA Video Connect for evidence-based psychotherapies for anxiety and depression in the Veterans Healthcare Administration." They assessed successes and challenges associated with taking a system that was already in place that expanded rapidly implementation after COVID-19 hit, using administrative data and qualitative interviews with providers. Within 1 year of the initiative, providers experienced with telebehavioral health increased from 15 to $85 \%$, and telehealth appointments tripled. Barriers to successful implementation included administrative challenges like appointment scheduling, licensing, and supervision issues and concerns regarding care such as determining clinical appropriateness for telehealth. Not unlike many other programs, successful strategies for increasing the use of telebehavioral health services included a champion-based model plus system-level initiatives mandating providers to test the Video-Connect system with Veterans.

Finally, Bechtel et al. examined "Care manager perspectives on integrating an mHealth app system into clinical workflows: a mixed methods study" with patients with posttraumatic stress disorder and/or bipolar disorder in rural settings and care managers from 12 rural federally qualified health centers in 3 states. They acquired feedback on adoption, use, and impact on care of an Android-only mobile health system in a collaborative care program. Six themes were identified in two categories: Facilitating conditions (need for cross-platform and web availability for mobile systems, limits imposed by existing technological infrastructure, inconsistencies, and redundancies between research and standard care workflows, habit as a minor theme) and clinical care (engagement and clinically disengaged app use, safety planning tool integration). They summarize with the recommendation that to maximize adoption, mobile systems should be accessible from multiple mobile platforms and developed in conjunction with research and existing clinical workflows.

\section{Section 2}

Section 2 shifts the focus onto educational interventions, competencies, and organizational leadership, and there are six interesting perspectives covered. Cruz et al. conducted "A survey of residents/fellows, program directors and faculty about telepsychiatry: clinical experience, interest, and views/concerns" using a cross-sectional survey sent through national organization Listservs. They had 270 respondents who had limited clinical experience with telepsychiatry but were very interested or interested in it. Surprisingly, trainees had concerns about being prepared for future practice, and all had concerns about connectivity, medico-legal, and clinical effectiveness with just aspects as diverse populations, psychosis, and emergencies with telepsychiatry. It was good to see that clinical experience with telepsychiatry-as little as $1-5 \mathrm{~h}$ though more substantially with $6-20 \mathrm{~h}$-increased interest and reduced concerns. They note that additional curricular interventions during training could build skills and confidence.

"A survey of child and adolescent fellows, faculty and program director perspectives about telepsychiatry: implications for clinical care and training" by Orchard et al. provides additional perspectives, using a similar design with a crosssectional survey. The respondents also had less clinical experience with telepsychiatry than adult counterparts and surprisingly trainees had less interest than faculty. Respondents were worried about the ability to do a physical exam, connectivity, medico-legal issues, fit for diverse populations, and loss of nonverbal cues. Those with clinical experience reported fewer concerns and more interest, suggesting that replicable, curricular interventions are essential — and faculty, too, need clinical experience with telepsychiatry.

Three papers by Hilty et al. deal with competencies. All three papers promoted skills as a way to ensure the quality of patient-centered care. They described measurable behaviors at three learner levels and case examples are provided on how to apply the competencies to care and to teach them with 
interactive versus didactic methods. They also offer suggestions on curricular methodologies, faculty development, and institutional practices - these things unify clinical, educational, and research missions and help create a positive e-culture for learning. "A scoping review to develop a framework of asynchronous technology competencies for psychiatry and medicine" included 86 papers and examined asynchronous clinical workflow, behavioral health professions' documents, and telepsychiatric and telebehavioral health competencies. They provide a framework for asynchronous technologies that covers patient care, medical/health knowledge, systems-based practice, practice-based learning and improvement, professionalism, interpersonal skills, and communication. Also addressed is an organizational change related to the major paradigm shift of in-person and synchronous care to include asynchronous technologies.

Hilty et al. also propose "A framework of sensor, wearable and remote patient monitoring competencies for clinical care and training: scoping review." They conclude that these new technologies integrate health, lifestyle, and clinical care, and contextually change the culture of care and training. Providers have more time for engagement, continuity of experience, and dynamic data for decision-making for both patients and clinicians, but this poses challenges like keeping up, getting education/training, and acquiring necessary skills. In "Mobile health and cultural competencies as a foundation for telehealth care: scoping review," the authors considered components of culturally competent, mobile health care, and approaches clinicians and systems have taken to implement care, train clinicians, and evaluate outcomes. There were 183 papers reviewed, although few discussed telehealth skills or competencies, mobile health, or cultural factors. They developed a set of cultural competencies, noting that we need a better understanding of how competencies may align or integrate with other competency areas such as integrated telehealth, rural health, and unique populations.

The final paper by Shoemaker et al. is entitled, "Flipping a child development lecture to active learning: Do the benefits justify the costs?" Medial schools are shifting to web-based and video technology rapidly, sometimes without being able to predict the impact of this change on learner outcomes. The objectives of this experiment were to (1) compare active learning methods versus traditional lecture for transmitting and retaining knowledge about child development, and (2) weigh whether the costs required to flip instruction are justified by learning gains. Active learning was less effective than lecture for knowledge acquisition and retention, as well as more expensive to do, but it promotes skill and attitudinal shifts, student satisfaction, and movement toward an institutional culture of interactive learning. Future research needs to evaluate replicable changes, consider direct and indirect costs and benefits, and weigh the scale of implementation.

\section{Conclusion}

In reviewing this compilation of papers, the guest editors had some key observations. The evidence base in this area is increasing dramatically, but still has gaps, with the preponderance of evidence and research on video-based delivery of care. The COVID pandemic has furthered the experience with video and telephone in a helpful way for most care participants. mHealth, apps, and wearable technologies will clearly be used increasingly in the future, but a significant amount of research still needs to be conducted to create a solid evidence base. Developing frameworks for the assessment of technologies as well as their impact on users and what competencies are required is critical, but we are far from having a national or international consensus on what this should entail, how they should be assessed, and how often they might need to be changed as newer technologies are developed - this is true for trainees and providers across behavioral health. There also has to be a balance between developing competencies indepth with a general set across technologies to be practical. We hope that the articles in the special issue will be of interest to a wide range of readers and inspire readers to further consider these important issues, conduct research, and advocate for broader adoption of training curriculums and core competencies.

Publisher's Note Springer Nature remains neutral with regard to jurisdictional claims in published maps and institutional affiliations. 